\title{
How did the COVID-19 pandemic affect clinical training of Malaysian dental students: final year students' perspectives
}

\author{
Yew, H.Z. ${ }^{1}$, Dom, T.N.M. ${ }^{1}$, Rani, H. ${ }^{1}$, Chanthirasekar, N.A.L. ${ }^{1}$, Yin, L.L. ${ }^{1}$, Bukhari, M. ${ }^{1}$, Xin, \\ L.K. ${ }^{1}$, Sabri, B.A.M. ${ }^{2}$, Rosdy, N.M.M.N.M. ${ }^{3}$, Esa, ${ }^{3}{ }^{3}$, Kacharaju, K.R. ${ }^{4}$, Andiesta, N.S. ${ }^{4}$, \\ Parolia, A. $^{5}$ \\ ${ }^{1}$ University Kebangsaan Malaysia, ${ }^{2}$ Faculty of Dentistry, Universiti Teknologi Mara (UiTM), ${ }^{3}$ Universiti \\ Teknologi Mara (UiTM), MAHSA University, ${ }^{5}$ International Medical University, Malaysia
}

\section{Background:}

The national lockdown due to the COVID-19 pandemic implemented by the Government has significantly affected the teaching and learning in dental schools. The reopening of dental school clinics with strict adherence to the standard operating procedures required major adaptations from all parties including students. This study aimed to assess the perspective of dental students on the challenges faced while undergoing clinical training during the COVID-19 pandemic.

\section{Methods:}

Ethical approval was obtained from the National University of Malaysia Ethics Committee (JEP-2020690). Data were collected from fifth-year dental students pursuing undergraduate programs in 13 dental schools in Malaysia using an online questionnaire. The questionnaire was pilot tested prior to distribution.

\section{Results:}

The study yielded a response rate of $42.4 \%(n=313)$. Female students dominated the total survey $(n=237,75.7 \%)$, while $63.2 \%(n=198)$ of responses were received from public universities. Almost all dental students ( $n=312,99.7 \%$ ) believed that the COVID-19 poses risks to dental practitioners. Majority of the students reported they have sufficient knowledge $(n=291,93.0 \%)$ and confidence $(n=249,79.6 \%)$ in performing their clinical duties. Moreover, most students have opined that they are prepared $(n=227$, $72.5 \%)$ and know ( $n=275,87.8 \%$ ) how to manage infected patients. Positive responses ( $n=275,87.9 \%)$ were received with regards to their schools' facilities in handling COVID-19. Both clinical supervisors and auxiliary staff were perceived to be able to support their training $(n=301,96.1 \%)$. Although a high number of students $(n=296,94.5 \%)$ were aware of the COVID-19 guidelines in dental practice, they $(n=276,88.2 \%)$ were overwhelmed by the available information regarding the pandemic. A significant proportion of students indicated that the pandemic has affected their ability to complete their clinical requirement $(n=277,88.5 \%)$ and their preparedness to be a dentist $(n=229,73.1 \%)$. Most respondents $(n=268,85.6 \%)$ expressed fear of getting infected from their patients and co-workers. They were also concerned of transmitting the infection to their families $(n=300,95.9 \%)$. Likewise, around $92 \%(n=248)$ of participants were afraid of getting quarantined from the infection. When in proximity with patients, 
$66.7 \%(n=209)$ students were nervous. Most students $(n=302,95.6 \%)$ reported seeing fewer patients in each clinical session and were anxious $(n=292,90 \%)$ if they were to provide treatment to patients suspected of being infected. Slightly more than half $(n-166,53.0 \%)$ of the students reported having financial impact due to COVID-19 but only $8.9 \%(n=36)$ of them would consider a career change.

\section{Conclusion:}

Despite the challenges faced by the profession, the graduating dental students appear to be highly selfassured in carrying out their clinical practice. Nevertheless, significant impact was felt on their readiness to become full-fledged dentists. Therefore, a supportive and holistic framework is pertinent to address students' overall well-being while getting through clinical training during an infectious disease outbreak, in preparation for their post-graduation independent practice.

Keywords: Dental Education 